\title{
Addition of Definitive Radiotherapy to Chemotherapy in Patients With Newly Diagnosed Metastatic Nasopharyngeal Cancer
}

\author{
Vivek Verma, MDa; Pamela K. Allen, $\mathrm{PhD}^{\mathrm{b}}$; Charles B. Simone II, MDc; Hiram A. Gay, MDd; \\ and Steven H. Lin, MD, $\mathrm{PhD}^{\mathrm{b}}$
}

\begin{abstract}
Background: Management of metastatic (M1) nasopharyngeal cancer (NPC) is controversial; data suggest high overall survival (OS) rates with definitive chemoradiotherapy (CRT). Herein, we evaluated OS in patients with M1 NPC undergoing chemotherapy alone versus CRT. Methods: The National Cancer Data Base was queried for M1 NPC cases. Patients undergoing no/unknown chemotherapy and/or with unknown/nondefinitive radiotherapy (RT) doses (<60 Gy) were excluded. Logistic regression analysis ascertained clinical factors associated with RT administration. Kaplan-Meier analysis evaluated OS between both cohorts; Cox proportional hazards modeling assessed factors associated with OS. Survival was then evaluated between matched populations using inverse-probability-weighted regression adjustment. OS between groups was also measured in patients surviving $\geq 1$ and $\geq 3$ years to address bias from poor-prognostic subsets (eg, widely disseminated disease), and those receiving CRT $\leq 30$ and $\leq 60$ days of each other (surrogates for concurrent CRT) versus $>30$ and $>60$ days (sequential) of each other. Results: Of 555 patients, 296 (53\%) received chemotherapy alone and 259 (47\%) underwent CRT. Patients undergoing CRT more often had private insurance $(P=.001)$ and lived in areas with higher education levels $(P=.028)$. Median OS in the chemotherapy-only and CRT cohorts were 13.7 and 25.8 months, respectively $(P<.001)$; differences persisted between matched populations $(P<.001)$. On multivariate analysis, receipt of additional RT independently predicted for improved OS $(P<.001)$. OS differences between cohorts remained apparent when evaluating patients surviving for $\geq 1(P<.001)$ and $\geq 3(P=.002)$ years. Patients who received concurrent or sequential CRT displayed improved OS over those receiving chemotherapy alone, for both the 30-day $(P<.001)$ and 60-day cutoffs $(P<.001)$. Conclusions: Patients with M1 NPC undergoing definitive RT and chemotherapy experienced higher survival than those receiving chemotherapy alone. Risk stratification and patient selection for such combined modality interventions is critical.
\end{abstract}

Although nasopharyngeal carcinoma (NPC) is relatively uncommon in the United States, it is endemic in East Asia, and is thus a global public health priority. ${ }^{1}$ Although NPC generally has a higher proclivity for distant metastases than other head and neck cancers, the incidence of metastatic (M1) disease at diagnosis is relatively rare, estimated to be $4 \%$ to $6 \% .^{2-5}$ However, 5 -year survival for M1 NPC has been quoted at approxi-

From ${ }^{a}$ Department of Radiation Oncology, University of Nebraska Medical Center, Omaha, Nebraska; 'Department of Radiation Oncology, University of Texas MD Anderson Cancer Center, Houston, Texas; 'Department of Radiation Oncology, University of Maryland Medical Center, Baltimore, Maryland; and ${ }^{\mathrm{D}}$ Department of Radiation Oncology, Washington University School of Medicine, St. Louis, Missouri.

Submitted March 28, 2017; accepted for publication June 27, 2017.

Dr. Lin has disclosed that he has received research funding from Elekta, STCube Pharmaceuticals, Peregrine, and Roche/Genentech; has served mately $20 \%$, a relatively high figure compared with other M1 malignancies. ${ }^{5}$ Moreover, a median overall survival (OS) of 2 to 3 years can be achieved for select patients with oligometastatic M1 NPC receiving palliative chemotherapy and/or those with good response to systemic therapy. ${ }^{6-8}$

In light of these observations, there has been intense debate questioning the validity of an all-encompassing

as a consultant for AstraZeneca; and has received honorarium from US Oncology and ProCure. The remaining authors have disclosed that they have no financial interests, arrangements, affiliations, or commercial interests with the manufacturers of any products discussed in this article or their competitors.

Correspondence: Steven H. Lin, MD, PhD, The University of Texas MD Anderson Cancer Center, Department of Radiation Oncology, 1515 Holcombe Boulevard, Unit 097, Houston, TX 77030.

E-mail: shlin@mdanderson.org 
"M1" or "stage IVC" label describing this heterogeneous patient population, and whether a subset of these patients could benefit from local therapy in addition to standard-of-care chemotherapy. ${ }^{9}$ This is in accord with the rapidly emerging oncologic concept of using local therapy for limited M1 disease (oligometastases). ${ }^{10-13}$ Implications for NPC are substantial, because distant M1 disease is generally more limited for NPC than for other solid malignancies, and just one to a few metastases occur in more than half of patients with M1 NPC. ${ }^{14}$ As a result, there has been increasing interest in whether administration of definitive chemoradiotherapy (CRT) for select patients with M1 NPC improves prognosis. ${ }^{15-20}$

However, this issue remains currently unresolved, with no consensus to date. ${ }^{21}$ The National Cancer Data Base (NCDB) provides a unique resource with which to address these novel and clinically important issues in a relatively uncommon population, and it has proven great utility for similar impactful studies in other neoplasms. ${ }^{22}$ This study compared patients with M1 NPC receiving chemotherapy alone versus CRT. We aimed to evaluate national practice patterns and the clinical factors associated with receiving combined modality therapy (CMT); to assess OS between groups, including factors independently associated with OS; and to determine the effect of concurrent versus sequential CRT on OS in CMT.

\section{Methods}

The NCDB is a joint project of the Commission on Cancer (CoC) of the American College of Surgeons and the American Cancer Society, which consists of de-identified information regarding tumor characteristics, patient demographics, and patient survival for approximately $70 \%$ of the US population. ${ }^{23-37}$ The NCDB contains information not included in the SEER database, including details regarding use of systemic therapy. The data used in the study were derived from a de-identified NCDB file, comprising all newly diagnosed cases by each CoC-accredited facility. The American College of Surgeons and the CoC have not verified and are neither responsible for the analytic or statistical methodology used, nor the conclusions drawn from these data by the investigators. Because all patient information in the NCDB database is de-identified, this study was exempt from Institutional Review Board evaluation.

Inclusion criteria for this study were patients with newly diagnosed M1 NPC. Patients with unknown receipt of chemotherapy and/or radiotherapy (RT) were excluded, as were patients who did not undergo chemotherapy. For the purposes of this study, definitive RT referred to doses $\geq 60$ Gy; although $\geq 66$ Gy to gross disease is recommended, ${ }^{21}$ we allowed for approximately a $10 \%$ dose reduction (60 Gy) as the threshold for inclusion. Patients in the chemotherapy only group were allowed to have received $\leq 30$ Gy of RT $(n=47)$, consistent with standard palliative irradiation dosing. In accordance with the variables in NCDB files, information collected on each patient broadly included demographic, clinical, and treatment data.

Statistics were performed with STATA software (StataCorp Inc., College Station, TX). Tests were 2 -sided, with a threshold of $P<.05$ for statistical significance. First, univariable and multivariable logistic regression were used to determine which characteristics were associated with delivery of additional RT. Next, Kaplan-Meier survival analysis evaluated OS, defined as the interval between the date of diagnosis and the date of death, or censored at last contact. Univariate and multivariate Cox proportional hazards modeling were used to evaluate predictors of OS.

Subsequently, survival analysis was performed in a matched population through the use of inverseprobability-weighted regression adjustment with nearest neighbor matching (Table 1). Nearest neighbor matching estimators impute the missing potential outcome for each subject by using an average of the outcomes of similar subjects that receive the other treatment level. Similarity between subjects is based on a weighted function of the covariates for each observation. This technique was performed with a nearest neighbor match of up to 4 cases, although a minimum of 1 match is required for this technique. Data were assessed with a treatment effects analytic technique using survival time to provide potential outcome mean as a measure of the effect (measured in units of time). The measure was adjusted for the factors found statistically significant on Cox regression analysis. Matching was applied to the whole cohort and not on subsequent (eg, landmark) analyses owing to low sample sizes in the latter. 
Chemoradiotherapy in M1 Nasopharyngeal Cancer

\begin{tabular}{|c|c|c|c|c|c|}
\hline RT Status & Coefficient & $Z$ Value & $P>|z|$ & SE & $95 \% \mathrm{Cl}$ \\
\hline \multicolumn{6}{|c|}{ Average Treatment Effect } \\
\hline CRT vs chemo alone & 8.33 & 1.94 & .05 & 4.29 & -0.08 to 16.74 \\
\hline Chemo alone & 20.97 & 9.87 & $<.01$ & 2.12 & 16.81 to 25.14 \\
\hline \multicolumn{6}{|c|}{ Potential Outcome Means } \\
\hline CRT vs chemo alone & 20.74 & 9.56 & $<.01$ & 2.17 & 16.48 to 24.99 \\
\hline Chemo alone & 29.21 & 8.49 & $<.01$ & 3.44 & 22.47 to 35.95 \\
\hline
\end{tabular}

Abbreviation: Chemo, chemotherapy; CRT, chemoradiotherapy; RT, radiotherapy.

Next, landmark analysis was performed in order to assess survival differences between both groups among patients surviving $\geq 1$ and $\geq 3$ years, with the purpose of eliminating patients with widely disseminated disease who would not be expected to survive past a fixed amount of time. The 1-year threshold was chosen because it approximates the median OS of M1 NPC; the 3-year value was chosen because previously noted reports have demonstrated 3-year survival of oligometastatic disease. ${ }^{6-8}$

Lastly, in attempt to compare OS between patients receiving chemotherapy alone and concurrent versus sequential CRT, OS was analyzed in patients receiving chemotherapy and RT within 30 days (a surrogate for concurrent CRT) and $>30$ days (surrogate for sequential CRT) of each other. This was repeated using the 60-day threshold based on the estimated time of induction chemotherapy from existing literature. ${ }^{38}$ If information regarding this parameter was not reported, those patients were excluded from this specific analysis.

\section{Results}

A complete flow diagram of patient selection is provided in Figure 1. In total, 555 patients met study analysis criteria, and included 296 (53\%) patients receiving chemotherapy alone and 259 (47\%) undergoing CRT. Table 2 displays notable clinical characteristics of both cohorts. Following univariable analysis, multivariable analysis revealed several independent factors associated with receipt of RT. Patients undergoing CRT were more likely to have private insurance $(P<.001)$ and lived in areas with higher education levels $(P=.028)$. Paradoxically, those undergoing CRT were less likely to have higher incomes $(P=.005)$. Of note, age, year of diagnosis, comorbidity index, $\mathrm{T}$ stage, and $\mathrm{N}$ stage were not associated with likelihood of receiving RT (all P>.05).

Kaplan-Meier estimates comparing OS in the chemotherapy only and CRT groups are illustrated in Figure 2A. The 1-, 3-, and 5-year OS rates in the respective groups were $54 \%$ versus $72 \%, 21 \%$ versus $41 \%$, and $10 \%$ versus $34 \%$. This corresponded to median OS of 13.7 and 25.8 months, respectively $(P<.001)$.

In the overall cohort, there were several predictors of OS on univariate and multivariate analysis (supplemental eTable 1, available online with this article at JNCCN.org). Among others, independent predictors of poor OS included higher comorbidity index, well to moderately differentiated disease, and T4/TX stage (all $P<.05$ ). Of note, there did not seem to be obvious independent associations with $\mathrm{N}$ stage and several socioeconomic and demographic variables. However, delivery of RT was an independent

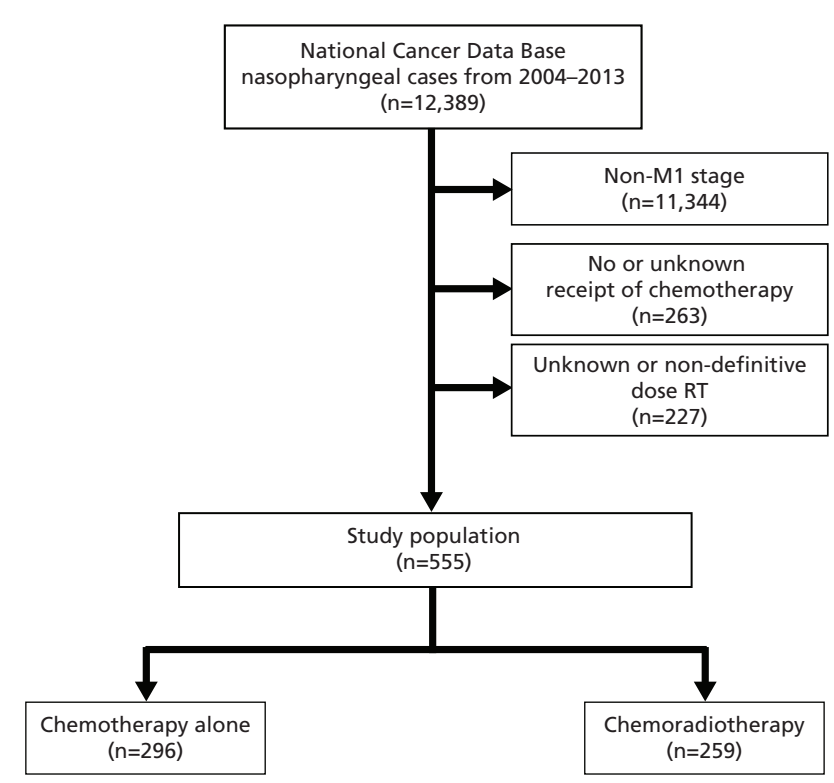

Figure 1. Patient selection diagram. 
Verma et al

\begin{tabular}{|c|c|c|c|c|c|c|}
\hline \multirow[b]{2}{*}{ Parameter, N (\%) } & \multirow{2}{*}{$\begin{array}{l}\text { Chemotherapy } \\
\text { Alone }(\mathrm{N}=296)\end{array}$} & \multirow[b]{2}{*}{ CRT (N=259) } & \multicolumn{2}{|c|}{ Univariate } & \multicolumn{2}{|c|}{ Multivariate $^{\mathrm{a}}$} \\
\hline & & & OR $(95 \% \mathrm{Cl})$ & $P$ Value & OR $(95 \% \mathrm{Cl})$ & $P$ Value \\
\hline Median age (range), $y$ & $56(18-90)$ & $54(18-87)$ & $1.00(0.99-1.01)$ & .706 & & \\
\hline \multicolumn{7}{|l|}{ Sex } \\
\hline Male & $222(75 \%)$ & $205(79 \%)$ & Ref & Ref & & \\
\hline Female & $74(25 \%)$ & $54(21 \%)$ & $0.79(0.53-1.18)$ & .247 & & \\
\hline \multicolumn{7}{|l|}{ Race } \\
\hline White & $142(48 \%)$ & $144(56 \%)$ & Ref & Ref & & \\
\hline Black & $57(19 \%)$ & $29(11 \%)$ & $0.50(0.30-0.83)$ & .007 & & \\
\hline Hispanic & $37(13 \%)$ & $21(8 \%)$ & $0.56(0.31-1.00)$ & .051 & & \\
\hline Other & $60(20 \%)$ & $65(25 \%)$ & $1.07(0.70-1.63)$ & .758 & & \\
\hline \multicolumn{7}{|l|}{ Insurance type } \\
\hline Private & $99(33 \%)$ & $142(55 \%)$ & $2.94(1.61-5.39)$ & $<.001$ & $2.84(1.52-530)$ & .001 \\
\hline Medicare & $80(27 \%)$ & $56(22 \%)$ & $1.44(0.75-2.74)$ & .271 & $1.34(0.69-2.60)$ & .381 \\
\hline Medicaid & $62(21 \%)$ & $37(14 \%)$ & $1.22(0.62-2.43)$ & .560 & $1.11(0.55-2.22)$ & .771 \\
\hline Other governmental & $6(2 \%)$ & $0(0 \%)$ & - & - & - & - \\
\hline Uninsured & $39(13 \%)$ & $19(7 \%)$ & Ref & Ref & Ref & Ref \\
\hline Unknown & $10(3 \%)$ & $5(2 \%)$ & $1.03(0.31-3.43)$ & .966 & $0.85(0.25-2.89)$ & .800 \\
\hline \multicolumn{7}{|l|}{ Annual income (USD) } \\
\hline$<\$ 48,000$ & $133(45 \%)$ & $126(49 \%)$ & Ref & Ref & Ref & Ref \\
\hline $2 \$ 48,000$ & $155(52 \%)$ & $128(49 \%)$ & $0.87(0.62-1.22)$ & .426 & $0.55(0.36-0.83)$ & .005 \\
\hline Unknown & $8(3 \%)$ & $5(2 \%)$ & - & - & - & - \\
\hline \multicolumn{7}{|l|}{ Location } \\
\hline Metropolitan & $244(82 \%)$ & $217(84 \%)$ & Ref & Ref & & \\
\hline Urban & $35(12 \%)$ & $26(10 \%)$ & $0.84(0.49-1.43)$ & .513 & & \\
\hline Rural & $5(2 \%)$ & $3(1 \%)$ & $0.67(0.16-2.86)$ & .593 & & \\
\hline Unknown & $12(4 \%)$ & $13(5 \%)$ & $1.22(0.54-2.73)$ & .631 & & \\
\hline \multicolumn{7}{|l|}{ Facility type } \\
\hline Community & $124(42 \%)$ & $121(47 \%)$ & Ref & Ref & & \\
\hline Academic/Research-Integrated Network & $116(39 \%)$ & $108(42 \%)$ & $0.95(0.66-1.37)$ & .800 & & \\
\hline Unknown & $56(19 \%)$ & $30(12 \%)$ & - & - & & \\
\hline \multicolumn{7}{|l|}{ Facility location } \\
\hline New England & $9(3 \%)$ & $10(4 \%)$ & Ref & Ref & & \\
\hline Middle Atlantic & $40(14 \%)$ & $38(15 \%)$ & $0.86(0.31-2.33)$ & .760 & & \\
\hline South Atlantic & $43(14 \%)$ & $45(17 \%)$ & $0.94(0.35-2.54)$ & .906 & & \\
\hline East North Central & $33(11 \%)$ & $37(14 \%)$ & $1.01(0.37-2.79)$ & .986 & & \\
\hline East South Central & $20(7 \%)$ & $17(7 \%)$ & $0.77(0.25-2.32)$ & .636 & & \\
\hline West North Central & $15(5 \%)$ & $12(5 \%)$ & $0.72(0.22-2.34)$ & .585 & & \\
\hline West South Central & $23(8 \%)$ & $19(7 \%)$ & $0.74(0.25-2.20)$ & .593 & & \\
\hline Mountain & $11(4 \%)$ & $8(3 \%)$ & $0.65(0.18-2.36)$ & .517 & & \\
\hline Pacific & $47(16 \%)$ & $43(17 \%)$ & $0.82(0.31-2.22)$ & .701 & & \\
\hline Unknown & $55(19 \%)$ & $30(12 \%)$ & - & - & & \\
\hline
\end{tabular}

Statistically significant $P$ values are in bold. Percentages may not add up to $100 \%$ due to rounding.

Abbreviations: CRT, chemoradiotherapy; OR, odds ratio.

aMultivariate analysis contains factors present in the final model.

predictor of higher OS (hazard ratio [HR], 0.51; 95\% CI, $0.41-0.63 ; P<.001)$.

Next, patients in both cohorts (242/296 chemotherapy alone, 230/259 CRT) were matched for the aforementioned factors associated with OS. KaplanMeier estimates comparing OS in the matched populations (chemotherapy alone vs CRT) are displayed in Figure 2B. The 1-, 3-, and 5-year OS in the respective groups were $53 \%$ versus $73 \%, 21 \%$ versus $42 \%$, and $11 \%$ versus $34 \%$. This corresponded to median OS of 13.6 and 27.6 months, respectively $(P<.001)$, similar to the unmatched groups.

Thereafter, to assess the value of additional RT in patients with presumed limited M1 disease, who may be expected to survive for a more extended period, landmark survival analysis was performed (Figure 3). The purpose of landmark analysis herein was to specifically attempt to counteract the selection bias between groups and evaluate a relatively "purer" population of survivors. Among the patients who survived for $\geq 1$ year after diagnosis, receipt of CMT remained associated with improved OS, corresponding to a median OS of 26.6 versus 50.7 months $(P<.001)$. This was also true for the patients surviving $\geq 3$ years, with a median OS of 54.6 months versus not reached $(P=.002)$.

Lastly, we assessed the effect of CRT in the concurrent versus sequential settings, while comparing with patients receiving chemotherapy alone (Figure 4). A threshold of receiving chemotherapy and RT 
Chemoradiotherapy in M1 Nasopharyngeal Cancer

\begin{tabular}{|c|c|c|c|c|c|c|}
\hline \multirow[b]{2}{*}{ Parameter, N (\%) } & \multirow{2}{*}{$\begin{array}{l}\text { Chemotherapy } \\
\text { Alone }(\mathrm{N}=296)\end{array}$} & \multirow[b]{2}{*}{ CRT $(\mathrm{N}=259)$} & \multicolumn{2}{|c|}{ Univariate } & \multicolumn{2}{|c|}{ Multivariate $^{\mathrm{a}}$} \\
\hline & & & OR $(95 \% \mathrm{Cl})$ & $P$ Value & OR $(95 \% \mathrm{Cl})$ & $P$ Value \\
\hline Median distance to treating facility (range), miles & $8.2(0-2,546.3)$ & $8.5(0-571.3)$ & $1.00(1.00-1.00)$ & .325 & & \\
\hline \multicolumn{7}{|l|}{ Year of diagnosis } \\
\hline $2004-2008$ & $120(41 \%)$ & $119(46 \%)$ & Ref & Ref & & \\
\hline $2009-2013$ & $176(59 \%)$ & $140(54 \%)$ & $0.80(0.57-1.12)$ & .200 & & \\
\hline \multicolumn{7}{|l|}{ Adults in zip code without high school diploma (\%) } \\
\hline$\geq 21 \%$ & $95(32 \%)$ & $56(22 \%)$ & Ref & Ref & & \\
\hline $13 \%-20.9 \%$ & $89(30 \%)$ & $81(31 \%)$ & $1.54(0.99-2.41)$ & .057 & & \\
\hline $7 \%-12.9 \%$ & $68(23 \%)$ & $77(30 \%)$ & $1.92(1.21-3.06)$ & .006 & & \\
\hline$<7 \%$ & $36(12 \%)$ & $40(15 \%)$ & $1.88(1.08-3.29)$ & .026 & & \\
\hline Unknown & $8(3 \%)$ & $5(2 \%)$ & - & - & - & - \\
\hline \multicolumn{7}{|l|}{ Charlson-Deyo score } \\
\hline 0 & $232(78 \%)$ & $222(86 \%)$ & Ref & Ref & & \\
\hline 1 & $49(17 \%)$ & $28(11 \%)$ & $0.60(0.36-0.98)$ & .043 & & \\
\hline$\geq 2$ & $15(5 \%)$ & $9(3 \%)$ & $0.63(0.27-1.46)$ & .28 & & \\
\hline \multicolumn{7}{|l|}{ Tumor grade } \\
\hline Well-differentiated & $5(2 \%)$ & $3(1 \%)$ & Ref & Ref & & \\
\hline Moderately differentiated & $25(8 \%)$ & $22(8 \%)$ & $1.47(0.31-6.85)$ & .626 & & \\
\hline Poorly differentiated & $117(40 \%)$ & $105(41 \%)$ & $1.50(0.35-6.41)$ & .588 & & \\
\hline Undifferentiated & $62(21 \%)$ & $61(24 \%)$ & $1.64(0.38-7.16)$ & .511 & & \\
\hline Unknown & $87(29 \%)$ & $68(26 \%)$ & $1.30(0.30-5.64)$ & .724 & & \\
\hline \multicolumn{7}{|l|}{ T stage } \\
\hline 0 & $1(0 \%)$ & $2(1 \%)$ & & & & \\
\hline 1 & $59(20 \%)$ & $43(17 \%)$ & & & & \\
\hline 2 & $42(14 \%)$ & $46(18 \%)$ & $1.46(0.83-2.58)$ & .193 & & \\
\hline 3 & $52(18 \%)$ & $52(20 \%)$ & $1.33(0.77-2.30)$ & .301 & & \\
\hline 4 & $81(27 \%)$ & $96(37 \%)$ & $1.58(0.97-2.57)$ & .065 & & \\
\hline Unknown & $61(21 \%)$ & $20(8 \%)$ & $0.44(0.23-0.83)$ & .011 & & \\
\hline \multicolumn{7}{|l|}{$\mathrm{N}$ stage } \\
\hline 0 & $25(8 \%)$ & $21(8 \%)$ & Ref & Ref & & \\
\hline 1 & $68(23 \%)$ & $60(23 \%)$ & $1.05(0.53-2.07)$ & .887 & & \\
\hline 2 & $96(32 \%)$ & $92(36 \%)$ & $1.14(0.60-2.18)$ & .690 & & \\
\hline 3 & $60(20 \%)$ & $45(17 \%)$ & $0.89(0.44-1.79)$ & .750 & & \\
\hline Unknown & $47(16 \%)$ & $41(16 \%)$ & $1.04(0.51-2.12)$ & .918 & & \\
\hline
\end{tabular}

Statistically significant $P$ values are in bold. Percentages may not add up to $100 \%$ due to rounding.

Abbreviations: CRT, chemoradiotherapy; OR, odds ratio.

aMultivariate analysis contains factors present in the final model.

within 30 days was first used to denote concurrent CRT, with $>30$ days signifying sequential CRT. Using this 30-day cutoff, both sets of patients receiving CRT demonstrated similar OS and both displayed superior OS over chemotherapy alone $(P<.001)$. The analysis was then repeated using a 60-day threshold, which led to similar findings $(P<.001)$.

\section{Discussion}

Although the management of M1 NPC remains controversial, our analysis suggests that a certain proportion of patients may benefit from CMT. Although this analysis cannot address which patients are specifically most suited for receiving additional local therapy, these data should ideally prompt prospective investigations regarding the utility of definitive management in well-selected patients with M1 NPC.

Although we were not able to ascertain why certain patients received CMT, we did observe significant socioeconomic disparities regarding its administration. Principally, patients with private insurance and/ or living in areas with higher education levels were more likely to undergo CRT, although it is discordant that patients with higher income were less likely. The same was not true regarding patient/tumor-specific factors (eg, age, comorbidity index, $\mathrm{T} / \mathrm{N}$ stage) or practice patterns (eg, diagnosis in more recent years, treatment at academic centers, regional differences). Thus, it could be likely that socioeconomic causes, such as insurance coverage for definitive additional RT, were primary determinants of receiving CMT. Because a foremost goal of oncologic care is to deliver appropriate treatment to disparate populations, if our results are further validated, there could be major implications on payers and insurance coverage to administer definitive RT in select cases.

This analysis also showed a near-doubling of OS in patients undergoing CRT over chemotherapy alone (which persisted in matched cohorts), and the inde- 
Verma et al

A

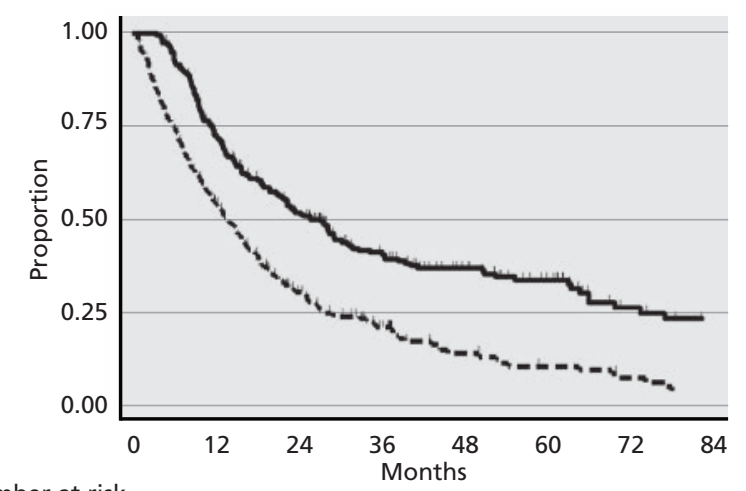

Number at risk

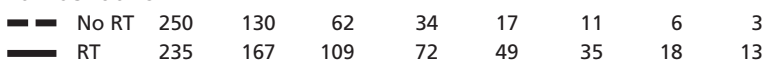

B

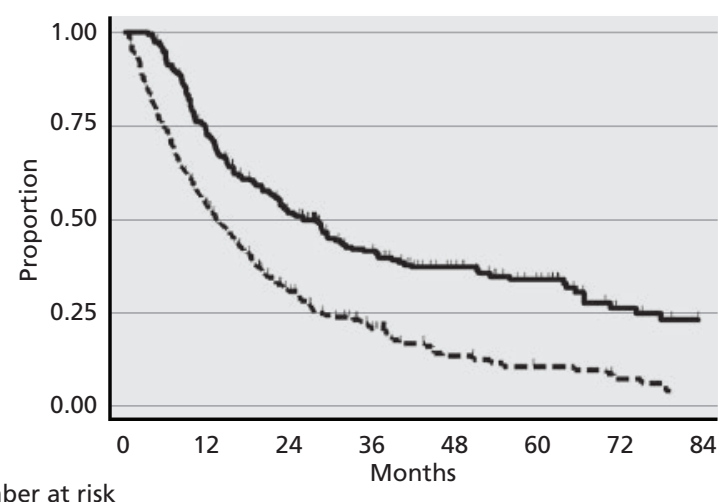

Number at risk

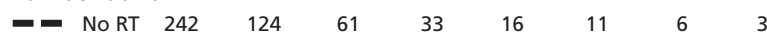

Figure 2. (A) Overall survival between populations receiving chemotherapy alone versus chemoradiotherapy $(P<.001)$. (B) Overall survival in the matched populations $(P<.001)$.

Abbreviation: $\mathrm{RT}$, radiotherapy.

pendent correlation with RT receipt and OS. The survival findings are similar to other institutional data; the 5 -year OS rate of $34 \%$ is comparable to the $39 \%$ rate observed by Tian et al. ${ }^{14}$ Additionally, a noteworthy finding on Kaplan-Meier OS curves was the relatively apparent "tail" associated with CMT, insinuating that a favorable prognostic subset of patients with M1 NPC could potentially experience long-term survival with aggressive oncologic therapy that includes high-dose local RT.

In any retrospective investigation, selection bias is clearly present to some degree, and thus caution is recommended when interpreting these and other analogous data. ${ }^{22}$ However, although both groups were statistically similar in terms of age and comor-

A

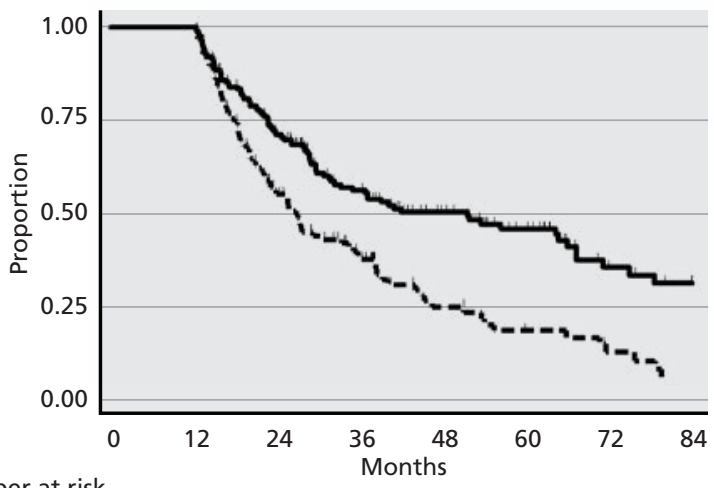

Number at risk

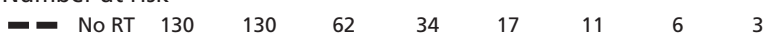

$\begin{array}{lrrrrrrrr}\text { RT } & 167 & 167 & 109 & 72 & 49 & 35 & 18 & 13\end{array}$ bidity index, a limitation of this and similar NCDB studies $^{22}$ is the lack of information regarding the number of metastatic lesions. Intuitively, RT may be more indicated in the oligometastatic setting, with multiple studies demonstrating that the prognosis of oligometastatic disease is superior to widely disseminated disease. ${ }^{15-20}$ In order to selectively analyze patients who could have oligometastatic NPC, landmark survival analysis was specifically designed to evaluate patients surviving $\geq 1$ and $\geq 3$ years after initial diagnosis in an attempt to use survival longevity as a surrogate for oligometastasis. Although not synonymous with oligometastatic cases, these improved prognostic subsets continued to gain OS benefits from definitive CMT. Conversely, the role of CRT in this analysis

\section{B}

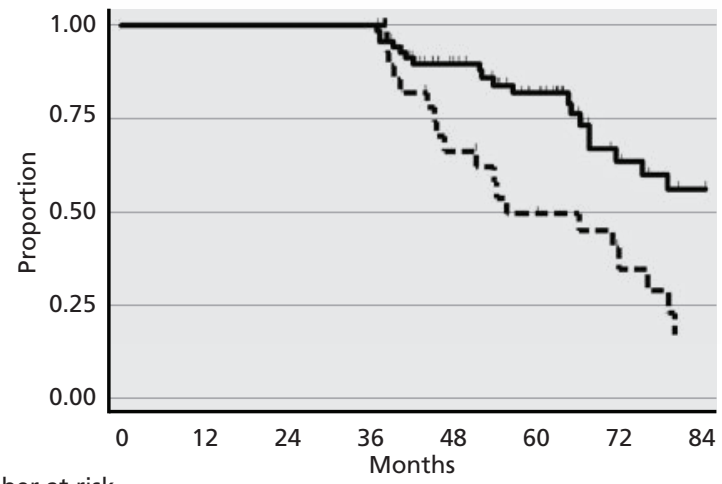

Number at risk

\begin{tabular}{|c|c|c|c|c|c|c|c|}
\hline No RT & 34 & 34 & 34 & 34 & 17 & 11 & 6 \\
\hline RT & 72 & 72 & 72 & 72 & 49 & 35 & 18 \\
\hline
\end{tabular}

Figure 3. Overall survival between cohorts receiving chemotherapy alone versus chemoradiotherapy $(A)$ in patients surviving for $\geq 1$ year $(P<.001)$, and $(B)$ in those surviving for $\geq 3$ years $(P=.002)$.

Abbreviation: $\mathrm{RT}$, radiotherapy. 
A

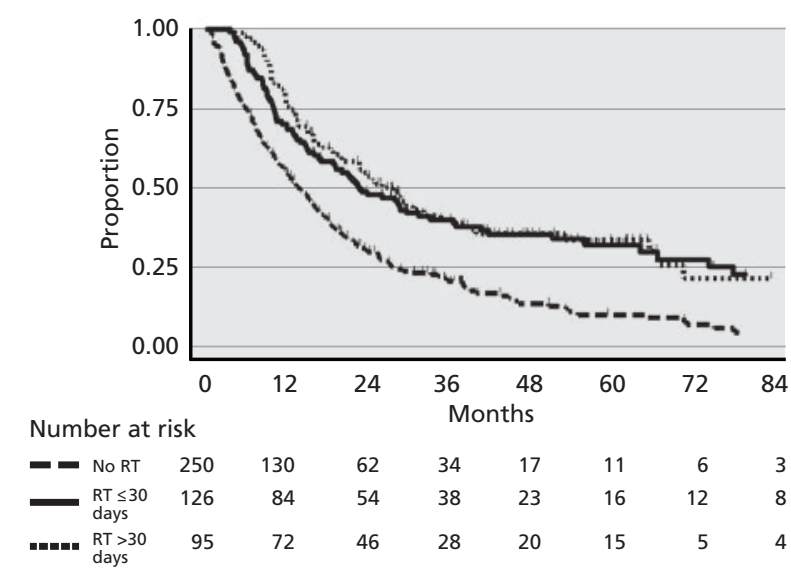

B

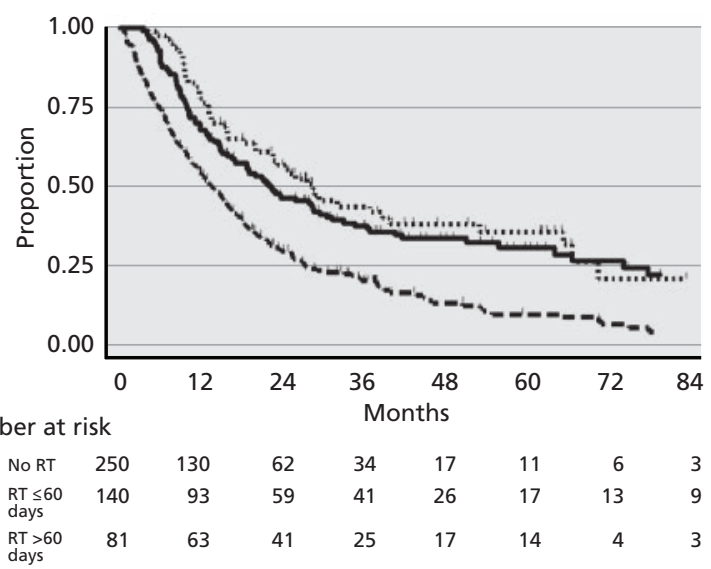

Figure 4. Comparison of overall survival in patients receiving chemotherapy alone versus chemoradiotherapy (A) within 30 days of each other versus chemoradiotherapy $>30$ days apart $(P<.001)$, and $(B)$ within 60 days of each other versus chemoradiotherapy $>60$ days apart $(P<.001)$.

Abbreviation: RT, radiotherapy.

was less clear in patients with poorer prognoses, many of whom presumably could have had more extensive M1 disease. Thus, moving forward, there should be an increasing risk stratification focus within the inherently heterogeneous M1 NPC cohort. Reports have alluded to several disease-specific factors that confer worse prognoses in M1 NPC, including greater quantity of metastatic lesions, delivery of $\leq 4$ chemotherapy cycles, liver metastases, specific gene panel markers, and Epstein-Barr virus (EBV) DNA levels. ${ }^{19,39-42}$ Taken together, although subdivision of the M1 category was not performed in the recent AJCC Cancer Staging Manual (8th edition), there is now mounting evidence of heterogeneity within M1 NPC that supports consideration of individualized, or personalized, treatment. ${ }^{43}$ Although our investigation does not imply causation between CMT and OS, it does underscore the critical importance of careful patient selection, especially in light of the amplified toxicities in patients receiving CMT. ${ }^{44-46}$

Herein, we recognized that delivery of CRT could follow 2 patterns: (1) receipt of chemotherapy alone, followed by presumed interval assessment of tumor response, and subsequently definitive RT; or (2) administration of upfront concurrent CRT. Using cutoff values of 30 and 60 days as a surrogate for concurrent versus sequential CRT, OS curves were similar in both the sequential and concurrent CRT cohorts, and both were statistically superior to the chemotherapy only survival. This may broaden the potential use of CMT in select patients with M1 NPC based on risk stratification; this includes the option to deliver CRT concurrently in patients with a clearly favorable prognosis, while examining disease response in more borderline cases and directing subsequent management thereafter. However, it is acknowledged that in this and similar studies ${ }^{22}$ there may be another layer of selection bias when evaluating such treatment paradigms. In patients with poor response after initial chemotherapy, RT may be withheld, and thus cases with poor biology would be lumped into the chemotherapy alone group, thereby adding a potential confounding factor. Conversely, however, prior reports have suggested that response to initial chemotherapy predicts response to RT in patients with head and neck malignancies. ${ }^{47,48}$ Regardless, our finding of improved OS in patients receiving concurrent, upfront CRT still illustrates that CMT may be efficacious in select patient subsets. Moreover, although the role of induction chemotherapy is controversial in light of previous $^{49}$ and recent ${ }^{22}$ data (of which the M1 subset has been virtually unrepresented), delivery of sequential chemotherapy and RT may be advantageous in these patients in light of similar survival observed herein while reducing the risk of toxicities seen with concurrent CRT, but additional prospective work is needed to determine the optimal temporal sequencing of CRT for M1 NPC.

Although the NCDB provides a unique platform with which to study this important clinical question, this investigation is not without limitations. First, NCDB studies are inherently retrospective with selection biases as noted earlier and elsewhere. ${ }^{22}$ Second, the NCDB does not keep track of several noteworthy vari- 
ables, such as tumor volume, location and number of metastatic lesions (and treatment thereof), EBV status (thus having more limited applicability to other regions worldwide), and smoking history. Third, the NCDB does not allow for an assessment of subsequent lines of treatment (eg, adjuvant and/or salvage systemic therapy, including targeted agents or immunotherapy), which could influence OS. Fourth, the NCDB does not provide details such as RT field design/volumes/techniques, specific chemotherapy type, or other end points, such as tolerance of therapy (including premature cessation of chemotherapy and/or RT), cancer-specific survival, and locoregional control. This includes the number of chemotherapy cycles (or induction, if given), because delivery of $\leq 4$ cycles correlates with worse prognosis ${ }^{39-42}$; hence, it is possible that patients in the chemotherapy only group received palliative chemotherapy in the form of fewer cycles and/or lower doses. Lastly, some patients classified as chemotherapy alone may have been treated with RT in an attempt to definitively control their disease. We attempted to minimize this bias by allowing patients receiving $\leq 30$ Gy to be categorized in the "chemotherapy only" group but used a cutoff dose for definitive RT of $\geq 60 \mathrm{~Gy}$. Although modest differences in the selected dose cut points are not likely to impact
OS, death from complications of poor CRT tolerance can never be excluded.

\section{Conclusions}

Using the NCDB, this contemporary analysis analyzed patients with M1 NPC receiving CRT versus chemotherapy alone. Socioeconomic factors (eg, private insurance, residence among higher-educated individuals) associated with the delivery of additional RT are described. Patients undergoing CRT experienced improved survival than those receiving chemotherapy alone, which was also present in matched cohorts. Receipt of RT independently predicted for increased survival. Among patients surviving for extended time periods, undergoing RT remained associated with improved OS. Lastly, using 2 different cutoff values to signify concurrent versus sequential CRT, both approaches displayed similarly higher OS over chemotherapy alone. Taken together, these hypothesis-generating data raise the need for prospective assessments regarding the use of local therapy in select M1 NPC cases. In the absence of prospective data, these results also underscore the crucial necessity to carefully select patients for definitive CRT.

\section{References}

1. Wei WI, Sham JS. Nasopharyngeal carcinoma. Lancet 2005;365:20412054.

2. Ahmad A, Stefani S. Distant metastases of nasopharyngeal carcinoma: a study of 256 male patients. J Surg Oncol 1986;33:194-197.

3. Lee AW, Poon YF, Foo W, et al. Retrospective analysis of 5037 patients with nasopharyngeal carcinoma treated during 1976-1985: overall survival and patterns of failure. Int J Radiat Oncol Biol Phys 1992;23:261-270.

4. Teo PM, Kwan WH, Lee WY, et al. Prognosticators determining survival subsequent to distant metastasis from nasopharyngeal carcinoma. Cancer 1996; 77:2423-2431.

5. Lee AW, Ng WT, Chan LK, et al. The strength/weakness of the AJCC/ UICC staging system (7th edition) for nasopharyngeal cancer and suggestions for future improvement. Oral Oncol 2012;48:1007-1013.

6. Chi KH, Chan WK, Cooper DL, et al. A phase II study of outpatient chemotherapy with cisplatin, 5-fluorouracil, and leucovorin in nasopharyngeal carcinoma. Cancer 1994;73:247-252.

7. Leong SS, Wee J, Rajan S, et al. Triplet combination of gemcitabine, paclitaxel, and carboplatin followed by maintenance 5-fluorouracil and folinic acid in patients with metastatic nasopharyngeal carcinoma. Cancer 2008;113:1332-1337.

8. Chua DT, Yiu HH, Seetalarom K, et al. Phase II trial of capecitabine plus cisplatin as first-line therapy in patients with metastatic nasopharyngeal cancer. Head Neck 2012;34:1225-1230.

9. Chan OS, Ngan RK. Individualized treatment in stage IVC nasopharyngeal carcinoma. Head Neck 2014;50:791-797.

10. Iyengar $\mathrm{P}$, Kavanagh $\mathrm{BD}$, Wardak $\mathrm{Z}$, et al. Phase II trial of stereotactic body radiation therapy combined with erlotinib for patients with limited but progressive metastatic non-small-cell lung cancer. J Clin Oncol 2014;32:3824-3830.

11. Gomez DR, Blumenschein Jr GR, Lee JJ, et al. Local consolidative therapy versus maintenance therapy or observation for patients with oligometastatic non-small-cell lung cancer without progression after firstline systemic therapy: a multicentre, randomised, controlled, phase 2 study Lancet Oncol 2016;17:1672-1682.

12. Xanthopoulos EP, Handorf E, Simone CB II, et al. Definitive dose thoracic radiation therapy in oligometastatic non-small cell lung cancer: hypothesis-generating study. Pract Radiat Oncol 2015;5:e355-363.

13. Baumann BC, Nagda SN, Kolker JD, et al. Efficacy and safety of stereotactic body radiation therapy for the treatment of pulmonary metastases from sarcoma: a potential alternative to resection. J Surg Oncol 2016;114:65-69.

14. Tian $Y$, Zou W, Xiao W, et al. Oligometastases in AJCC stage IVC nasopharyngeal carcinoma: a subset with better overall survival. Head Neck 2016;38:1152-1157.

15. Setton J, Wolden S, Caria N, Lee N. Definitive treatment of metastatic nasopharyngeal carcinoma: report of 5 cases with review of literature. Head Neck 2012;34:753-757.

16. Chen M, Jiang R, Guo L, et al. Locoregional radiotherapy in patients with distant metastases of nasopharyngeal carcinoma at diagnosis. Chin J Cancer 2013;32:604-613.

17. Zeng L, Tian Y, Huang Y, et al. Retrospective analysis of 234 nasopharyngeal carcinoma patients with distant metastasis at initial diagnosis: therapeutic approaches and prognostic factors. PLoS One 2014;9:e108070.

18. Hu S, He $X$, Dong $M$, et al. Systemic chemotherapy followed by locoregional definitive intensity-modulated radiation therapy yields prolonged survival in nasopharyngeal carcinoma patients with distant metastasis at initial diagnosis. Med Oncol 2015;32:224.

19. Shen L, Wang S, Xie G, et al. Subdivision of $M$ category for nasopharyngeal carcinoma with synchronous metastasis: time to expand the M categorization system. Chin J Cancer 2015;34:40.

20. Dandona MA, Morgensztern D, Auethavekiat V, et al. Survival for nasopharyngeal cancer with distant metastatic disease at presentation. J Clin Oncol 2009;27(Suppl):e17004

21. Pfister DG, Spencer S, Adelstein D, et al. NCCN Clinical Practice Guidelines in Oncology for Head and Neck Cancers, version 2.2016. 
Accessed December 26, 2016. To view the most recent version of these guidelines, visit NCCN.org.

22. Rusthoven CG, Jones BL, Flaig TW, et al. Improved survival with prostate radiation in addition to androgen deprivation therapy for men with newly diagnosed metastatic prostate cancer. J Clin Oncol 2016;34:2835-2842.

23. Bilimoria K, Stewart A, Winchester D, Ko C. The National Cancer Data Base: a powerful initiative to improve cancer care in the United States. Ann Surg Oncol 2008;15:683-690.

24. Bott MJ, Patel AP, Verma V, et al. Patterns of care in hilar node-positive (N1) non-small cell lung cancer: a missed treatment opportunity? J Thorac Cardiovasc Surg 2016;151:1549-1558.

25. Stahl JM, Corso CD, Verma V, et al. Trends in stereotactic body radiation therapy for stage I small cell lung cancer. Lung Cancer 2017;103:11-16.

26. Haque W, Verma V, Butler EB, Teh BS. Patterns of care and outcomes of multi-agent versus single-agent chemotherapy as part of multimodal management of low grade glioma. J Neurooncol 2017;133:369-375.

27. Haque W, Verma V, Butler EB, Teh BS. National practice patterns and outcomes for T4b urothelial cancer of the bladder [published online ahead of print September 6, 2017]. Clin Genitourin Cancer 2017, doi: 10.1016/j. clgc.2017.08.013.

28. Moreno AC, Verma V, Hofstetter WL, et al. Patterns of care and treatment outcomes of elderly patients with stage I esophageal cancer: analysis of the National Cancer Data Base. J Thorac Oncol 2017;12:1152-1160.

29. McMillan MT, Ojerholm E, Verma V, et al. Radiation treatment time and overall survival in locally advanced non-small cell lung cancer. Int J Radiat Oncol Biol Phys 2017;98:1142-1152.

30. Verma V, Ryckman JM, Simone CB II, Lin C. Patterns of care and outcomes with the addition of chemotherapy to radiation therapy for stage I nasopharyngeal cancer [published online ahead of print July 19, 2017]. Acta Oncol 2017, doi: 10.1080/0284186X.2017.1351039.

31. Verma V, Ahern CA, Berlind CG, et al. National Cancer Data Base report on pneumonectomy versus lung-sparing surgery for malignant pleural mesothelioma. J Thorac Oncol 2017;12:1704-1714.

32. Haque $\mathrm{W}$, Verma V, Fakhreddine M, et al. Addition of chemotherapy to definitive radiotherapy for IB1 and IIA1 cervical cancer: analysis of the National Cancer Data Base. Gynecol Oncol 2017;144:28-33.

33. Verma V, McMillan MT, Grover S, et al. Stereotactic body radiation therapy and the influence of chemotherapy on overall survival for large ( 25 centimeter) non-small cell lung cancer. Int J Radiat Oncol Biol Phys 2017;97:146-154.

34. Haque W, Verma V, Butler EB, Teh BS. Definitive chemoradiation at high volume facilities is associated with improved survival in glioblastoma. J Neurooncol 2017;135:173-181.

35. Haque W, Verma V, Butler EB, Teh BS. Radical cystectomy versus chemoradiation for musle-invasive bladder cancer: impact of treatment facility and sociodemographics. Anticancer Res 2017;37:5603-5608.

36. Haque W, Verma V, Butler EB, Teh BS. Radiation dose in neoadjuvant chemoradiation therapy for esophageal cancer: patterns of care and outcomes from the National Cancer Data Base [published online ahead of print]. J Gastrointest Oncol 2017, doi: 10.21037/jgo.2017.09.12.

37. Verma V, Simone CB, Lin C. Human papillomavirus and nasopharyngeal cancer [published online ahead of print]. Head Neck 2017; doi: 10.1002/ hed.24978.

38. Sun $\mathrm{Y}, \mathrm{Li}$ W, Chen $\mathrm{N}$, et al. Induction chemotherapy plus concurrent chemoradiotherapy versus concurrent chemoradiotherapy alone in locoregionally advanced nasopharyngeal carcinoma: a phase 3, multicentre, randomised controlled trial. Lancet Oncol 2016;17:1509-1520.

39. Ong YK, Heng DM, Chung B, et al. Design of a prognostic index score for metastatic nasopharyngeal carcinoma. Eur J Cancer 2003;39:1535-1541.

40. Toh CK, Heng D, Ong YK, et al. Validation of a new prognostic index score for disseminated nasopharyngeal carcinoma. Br J Cancer 2005;92:13821387.

41. Cao $X, \operatorname{Han} Y, H e L$, et al. Risk subset of the survival for nasopharyngeal carcinoma patients with bone metastases: who will benefit from combined treatment? Oral Oncol 2011;47:747-752.

42. Jin $Y$, Cai XY, Cai YC, et al. To build a prognostic score model containing indispensable tumour markers for metastatic nasopharyngeal carcinoma in an epidemic area. Eur J Cancer 2012;48:882-888.

43. Pan JJ, Ng WT, Zong JF, et al. Proposal for the 8th edition of the AJCC/ UICC staging system for nasopharyngeal cancer in the era of intensitymodulated radiotherapy. Cancer 2016;122:546-558.

44. Kwong DL, Sham JS, Au GK, et al. Concurrent and adjuvant chemotherapy for nasopharyngeal carcinoma: a factorial study. J Clin Oncol 2004;22:2643-2653.

45. Wee J, Tan EH, Tai BC, et al. Randomized trial of radiotherapy versus concurrent chemoradiotherapy followed by adjuvant chemotherapy in patients with American Joint Committee on Cancer/International Union against cancer stage III and IV nasopharyngeal cancer of the endemic variety. J Clin Oncol 2005;23:6730-6738.

46. Lee AW, Lau WH, Tung SY, et al. Preliminary results of a randomized study on therapeutic gain by concurrent chemotherapy for regionallyadvanced nasopharyngeal carcinoma: NPC-9901 Trial by the Hong Kong Nasopharyngeal Cancer Study Group. J Clin Oncol 2005;23:6966-6975.

47. Ensley JF, Jacobs JR, Weaver A, et al. Correlation between response to cisplatinum combination chemotherapy and subsequent radiotherapy in previously untreated patients with advanced squamous cell cancers of the head and neck. Cancer 1984;54:811-814.

48. Panis $X$, Coninx P, Nguyen TD, Legros M. Relation between responses to induction chemotherapy and subsequent radiotherapy in advanced or multicentric squamous cell carcinomas of the head and neck. Int J Radiat Oncol Biol Phys 1990;18:1315-1318.

49. Chua DT, Ma J, Sham JS, et al. Long-term survival after cisplatin-based induction chemotherapy and radiotherapy for nasopharyngeal carcinoma: a pooled data analysis of two phase III trials. J Clin Oncol 2005;23:11181124.

See JNCCN.org for supplemental online content. 